\title{
Generalized Pancharatnam-Berry Phase in Rotationally Symmetric Meta-Atoms
}

\author{
Xin Xie $\odot,{ }^{1,2,3}$ Mingbo Pu๑, ${ }^{1,2}$ Jinjin Jin, ${ }^{1}$ Mingfeng Xu, ${ }^{1,4}$ Yinghui Guo®, ${ }^{1,2}$ Xiong Li, ${ }^{1,2}$ \\ Ping Gao, ${ }^{1}$ Xiaoliang Ma, ${ }^{1,2}$ and Xiangang Luo $\odot^{1,2, *}$ \\ ${ }^{1}$ State Key Laboratory of Optical Technologies on Nano-Fabrication and Micro-Engineering, \\ Institute of Optics and Electronics, Chinese Academy of Sciences, Chengdu 610209, China \\ ${ }^{2}$ School of Optoelectronics, University of Chinese Academy of Sciences, Beijing 100049, China \\ ${ }^{3}$ Key Laboratory of Light Field Manipulation and Information Acquisition, Ministry of Industry and Information Technology, \\ and Shaanxi Key Laboratory of Optical Information Technology, School of Physical Science \\ and Technology, Northwestern Polytechnical University, Xi'an 710129, China \\ ${ }^{4}$ Division of Frontier Science and Technology, Institute of Optics and Electronics, \\ Chinese Academy of Sciences, Chengdu 610209, China
}

(Received 12 October 2020; accepted 31 March 2021; published 7 May 2021)

\begin{abstract}
Pancharatnam-Berry geometric phase has attracted enormous interest in subwavelength optics and electromagnetics during the past several decades. Traditional theory predicts that the geometric phase is equal to twice the rotation angle of anisotropic elements. Here, we show that high-order geometric phases equal to multiple times the rotation angle could be achieved by meta-atoms with highfold rotational symmetries. As a proof of concept, the broadband angular spin Hall effect of light and optical vortices is experimentally demonstrated by using plasmonic metasurfaces consisting of space-variant nanoapertures with $\mathrm{C} 2, \mathrm{C} 3$, and $\mathrm{C} 5$ rotational symmetries. The results provide a fundamentally new understanding of the geometric phase as well as light-matter interaction in nanophotonics.
\end{abstract}

DOI: 10.1103/PhysRevLett.126.183902

As a novel phase shift mechanism, the PancharatnamBerry geometric phase is a direct result of spin-orbit coupling of photons in structured materials, which has been widely used to control the light wave front and to design various types of functional metadevices known as geometric phase metasurfaces [1,2]. Different from other phase modulation approaches based on the variation of geometric sizes [3-5], these geometric phase metasurfaces are composed of arrays of meta-atoms in the form of rotated rectangles, ellipses, or more complex anisotropic patterns [6-12]. When circularly polarized light passes through such structures, the transmitted cross-polarized light will acquire a phase delay $\Phi$, which has a near linear dependence on the rotation angle $\varphi$ of each meta-atom, i.e., $\Phi= \pm 2 \varphi$, where the sign \pm corresponds to the incident left- and righthanded circular polarizations (LCP and RCP) $[13,14]$. This simple and robust phase control scheme offers straightforward routes for designing various phase-type optical elements, such as vortex plates [15-17], flat lenses [18-20], and metaholograms [21-24]. In principle, all previous methods to generate geometric phase rely on the polarization conversion enabled by anisotropic structures,

Published by the American Physical Society under the terms of the Creative Commons Attribution 4.0 International license. Further distribution of this work must maintain attribution to the author(s) and the published article's title, journal citation, and DOI. and subwavelength structures with $\geq 3$ rotational symmetries are thought to be isotropic and no geometric phase could be observed [25,26].

In nonlinear metasurfaces, the geometric phases can be written in a more general form. Under the illumination of circularly polarized fundamental wave, the transmitted nonlinear harmonic waves with the same or opposite helicity will carry the phase factors of $\pm(n-1) \varphi$ and $\pm(n+1) \varphi$, respectively [26-28], where $n$ is the order of harmonic generation. Since harmonic generations should satisfy the selection rules to ensure momentum conservation, structures with multiple rotational symmetry are required to generate the nonlinear signal [26].

In this Letter, we show for the first time that subwavelength structures with rotational symmetry $\geq 3$ can tailor the optical anisotropy owing to the lattice coupling effect, and further, high-order geometric phases that manifest as multiple times the rotation angle of the elements could be achieved in the linear optics regime. Several metasurfaces consisting of space-variant nanoapertures with $\mathrm{C} 3$ and C5 rotational symmetries are fabricated to demonstrate the wave front manipulation capacity. The results not only prove that the highly symmetric structures are anisotropic in symmetrically incompatible lattices, but also show that these structures can introduce geometric phases different from the previous relation of $\Phi= \pm 2 \varphi$. Since the geometric phase is imprinted on the fundamental wave, these effects are essentially different from the nonlinear phase for harmonic generations [26-29]. 

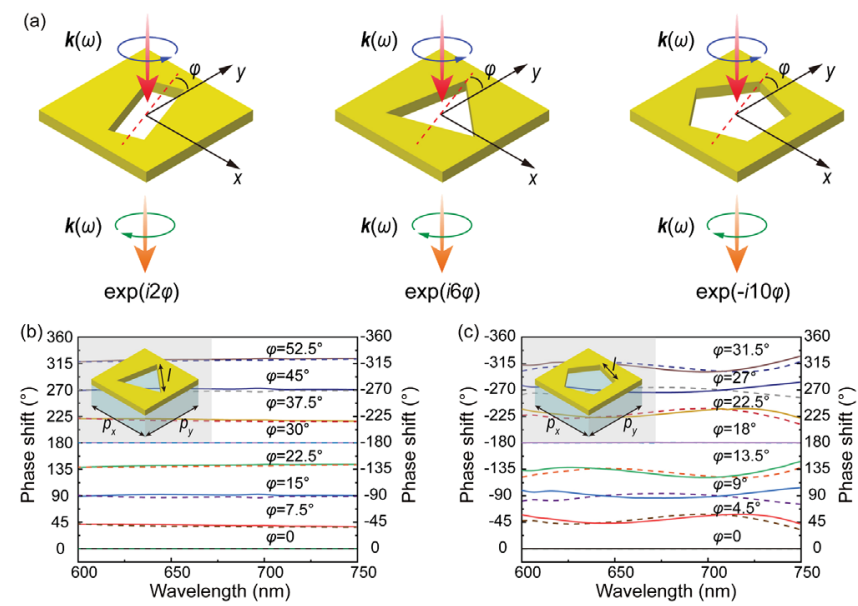

FIG. 1. (a) Illustration of meta-atoms consisting of rectangular, regular triangular, and regular pentagonal apertures. LCP illumination and RCP transmission are plotted. The apertures can rotate in the $x-y$ plane with an orientation angle of $\varphi$. (b),(c) Simulated phase shift of the transmitted cross-polarized light as a function of the orientation angle $\varphi$ of the regular (b) triangular and (c) pentagonal nanoapertures under LCP (solid lines, left axis) and RCP (dotted lines, right axis) illuminations. The insets illustrate the geometry of the designed meta-atoms, where 120-nm-thick gold films are deposited on $\mathrm{SiO}_{2}$ substrate. The periods and length are $p_{x}=p_{y}=200 \mathrm{~nm}$ and $l=160 \mathrm{~nm}$ for the triangular metaatoms, and $p_{x}=p_{y}=400 \mathrm{~nm}$ and $l=200 \mathrm{~nm}$ for the pentagonal meta-atoms.

Figure 1(a) shows three kinds of nanoapertures with differentfold rotational symmetries arranged in a square lattice. It is well known that the rectangular aperture with twofold rotational symmetry $(\mathrm{C} 2)$ can generate a geometric phase of $\pm 2 \varphi$ on the transmitted light with opposite handedness [1,2]. However, as shown in Figs. 1(b) and 1(c), for the regular triangular aperture with $\mathrm{C} 3$ rotational symmetry and the regular pentagonal aperture with C5 rotational symmetry, spin-dependent phases of about $\pm 6 \varphi$ and $\mp 10 \varphi$ are generated (see Sec. S1 of Supplemental Material for more details [30]).

The phase shifts shown in Figs. 1(b) and 1(c) have two notable properties. On the one hand, they are not completely opposite for LCP and RCP illuminations because the total phase shifts comprise both a spin-independent dynamic phase and a spin-dependent geometric phase (see Sec. S2 of Supplemental Material [30]). On the other hand, the phase shifts are exact multiple times of the orientation angle $\varphi$ for only some specific points. For the triangular (pentagonal) apertures under LCP illumination, when the angle $\varphi$ changes $30^{\circ}\left(18^{\circ}\right)$, there is a wavelengthindependent phase shift of $180^{\circ}\left(-180^{\circ}\right)$ for the crosspolarized wave, i.e., a geometric phase shift of $6 \varphi(-10 \varphi)$ are generated. By filtering out the dynamic phase, one could find that $\varphi=15^{\circ}\left(9^{\circ}\right)$ also belongs to the symmetric points, corresponding to a geometric phase shift of $90^{\circ}$ $\left(-90^{\circ}\right)$ for LCP illumination. For other intermediate rotation angles, the phase shifts are typically approximate values of the multiple relationships (see Sec. S2 of Supplemental Material [30]). Note that this approximate nature has also been observed in C2 structures [11,23,24].

The high-order geometric phase shifts can be understood by analyzing the rotational symmetry and the lattice coupling effect in the metasurfaces. Note that although both the lattice and single elements have high rotational symmetry $(\geq 3)$, the whole metasurface may be not isotropic. When the metasurface is anisotropic, there must be only two principal axes owing to the physical limitation of effective medium theory [31]. In this case, high-order geometric phase could be explained using the rotation of the effective principal axis (see Sec. S3 of Supplemental Material [30]).

For simplicity, we first analyze the case for meta-atoms in a square lattice. As illustrated in Fig. 2, for the $\mathrm{C} 1$ and $\mathrm{C} 2$ meta-atoms, the principal axis has an approximately equal rotation angle as the single meta-atom. For meta-atoms with three and higher rotational symmetry, however, there is a multiple relationship between the rotation angles of the principal axes and meta-atoms, as shown in Figs. 2(c)-2(f). Different from the cases of $\mathrm{C} 3$ and $\mathrm{C} 10$ structures, the rotation directions of the principal axes are opposite to that of single meta-atoms for C5 and C6 meta-atoms. When the C5 and C6 meta-atoms rotate by $9^{\circ}$ and $15^{\circ}$, both the principal axes rotate by $-45^{\circ}$. In principle, this unusual rotational characteristic cannot be easily figured out by only investigating the geometric features. Instead,

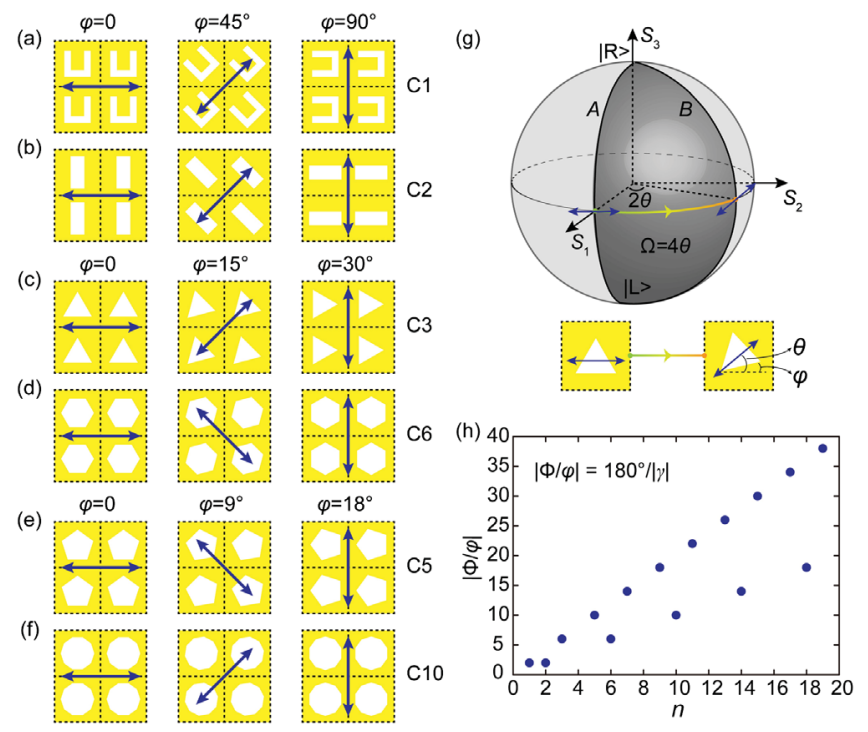

FIG. 2. Rotation dependence of the principal axis and metaatoms with (a) $\mathrm{C} 1$, (b) $\mathrm{C} 2$, (c) $\mathrm{C} 3$, (d) C6, (e) C5, and (f) $\mathrm{C} 10$ rotational symmetry. The double-headed arrows indicate the orientations of the principal array axis, which correspond to the evolution of the polarization states. (g) Schematic of polarization evolution on the Poincaré sphere for the triangular metaatom. (h) Absolute value of the coefficient $(|\Phi / \varphi|)$ of geometric phase for different rotational symmetries. 
numerical simulations of either the geometric phases or the transmission coefficients are required to determine the signs (see Secs. S2 and S4 of Supplemental Material [30]).

The novel geometric phase can also be understood via polarization evolution on the Poincaré sphere. As shown in Fig. 2(g), the north and the south poles of the sphere correspond to RCP $(|R\rangle)$ and LCP $(|L\rangle)$ states, respectively, and different azimuthal positions on the equator correspond to different linear polarization states (double-headed arrows). By taking advantage of the multifold relationship of $\theta$ and $\varphi$, the high-order geometric phase could be easily understood. For instance, when the $\mathrm{C} 3$ meta-atom has a rotation angle of $\varphi$, the principal axis will be rotated by $\theta$ and the light waves will undergo two different paths $A$ and $B$, resulting in a phase shift of $\Phi= \pm 2 \theta \approx \pm 6 \varphi$.

In the following, we extend the geometric phase theory to a more general case. For meta-atom with $n$-fold rotational symmetry placed in a square lattice, when the principal axis is rotated by $90^{\circ}$ (see Sec. S5 of Supplemental Material [30]), the meta-atom has a minimal rotation angle of

$$
\gamma=90^{\circ}-\frac{360^{\circ}}{n}\left[\frac{n}{4}\right]
$$

where [ ] is the nearest integer function. When $n$ is multiple of 4 , there is $\gamma=0$, implying that these elements have isotropic response. Considering that $\gamma$ is related to a rotation of $90^{\circ}$ for the principal axis, the general geometric phase could be written as

$$
\Phi=2 \times \frac{ \pm 90^{\circ}}{\gamma} \varphi
$$

Here, the sign of \pm can be determined by numerically investigating the rotating direction of the principal axis (see Sec. S4 of Supplemental Material [30]).

As illustrated in Fig. 2(h), the absolute coefficient of geometric phase for different rotational symmetry is divided into two branches based on the parity of the rotational symmetry. Consequently, Eq. (2) can be written in an alternative form based on the parity of symmetry (see Sec. S5 of Supplemental Material [30])

$$
\Phi= \begin{cases} \pm 2 n \varphi, & n \text { is odd } \\ \pm n \varphi, & n \text { is even }\end{cases}
$$

Similarly, the geometric phase of meta-atoms in the hexagonal lattice can be deduced with a bit more complex expressions (see Sec. S5 of Supplemental Material [30]). The geometric phase for both the square and hexagonal lattices are summarized in Table I, and the numerical results for C1-C10 meta-atoms are shown in Secs. S6 and S7 of Supplemental Material [30]. Note that the phase response can be observed for various apertures with the same local rotational symmetries as well as their Babinet-inverted structures [30]. According to these relationships, one can obtain a well-defined geometric phase with the desired gradient in the range of $0-2 \pi$ by choosing appropriate rotational symmetry of the meta-atoms and lattices.

One limit of our current results is the relatively low polarization conversion efficiency, which could be increased by adopting dielectric materials or reflective configuration (see Sec. S8 of Supplemental Material for details [30]). Note that the incident light needs to be at normal incidence, thus the rotational symmetry of the overall system is maintained. For light at oblique incidence, the symmetry would change and the phase responses may be deteriorated.

To demonstrate the concept of high-order geometric phase, three plasmonic gradient metasurfaces consisting of spatially varying triangular, Y-shaped, and pentagonal nanoapertures were designed to realize the angular spin Hall effect of light (SHEL) [37]. The phase gradient induced by a linear change of orientation with the coordinates produces a spin-dependent transverse wave vector. Thus, the gradient metasurfaces deflect LCP and RCP light to opposite directions. The sample fabrication and measurement procedures are provided in Sec. S9 of Supplemental Material [30]. As illustrated in Fig. 3(a), the supercells (dashed boxes) were designed to cover the optical phase from 0 to $2 \pi$ with 12 discrete levels that have equal phase jump. The cross-polarized electric fields $E_{\text {cross }}$ for circularly polarized incidence at a wavelength of $633 \mathrm{~nm}$ are filtered out and illustrated in Fig. 3(b). Under LCP incidence, the cross-polarized (RCP) light is deflected to the $x$ direction for the triangular and $\mathrm{Y}$-shaped metasurfaces while to the $-x$ direction for the pentagonal one. Under RCP incidence, reversed deflection angles can be observed. Figure 3(c) shows the simulated normalized far-field intensity distributions of the nanoapertures arrays for several different incident wavelengths. Excellent beam deflection can be observed in all the cases, agreeing well

TABLE I. Geometric phases introduced by meta-atoms with C1-C10 rotational symmetries in the square or hexagonal lattice under LCP and RCP illuminations.

\begin{tabular}{lccccccccccc}
\hline \hline Lattice & Polarization & $\mathrm{C} 1$ & $\mathrm{C} 2$ & $\mathrm{C} 3$ & $\mathrm{C} 4$ & $\mathrm{C} 5$ & $\mathrm{C} 6$ & $\mathrm{C} 7$ & $\mathrm{C} 8$ & $\mathrm{C} 9$ & $\mathrm{C} 10$ \\
\hline \multirow{2}{*}{ Square } & $\mathrm{LCP}$ & $2 \varphi$ & $2 \varphi$ & $6 \varphi$ & & $-10 \varphi$ & $-6 \varphi$ & $-14 \varphi$ & $18 \varphi$ & $10 \varphi$ \\
& $\mathrm{RCP}$ & $-2 \varphi$ & $-2 \varphi$ & $-6 \varphi$ & & $10 \varphi$ & $6 \varphi$ & $14 \varphi$ & $-18 \varphi$ & $-10 \varphi$ \\
Hexagonal & $\mathrm{LCP}$ & $2 \varphi$ & $2 \varphi$ & & $8 \varphi$ & $20 \varphi$ & & $14 \varphi$ & $8 \varphi$ & $-10 \varphi$ \\
& $\mathrm{RCP}$ & $-2 \varphi$ & $-2 \varphi$ & & $-8 \varphi$ & $-20 \varphi$ & $-14 \varphi$ & $-8 \varphi$ & $10 \varphi$ \\
\hline \hline
\end{tabular}




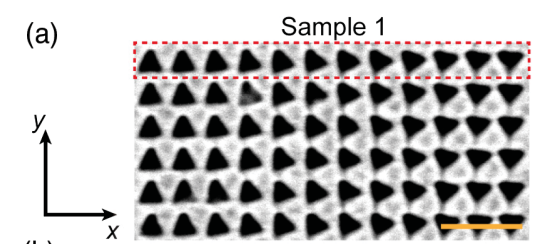

(b)

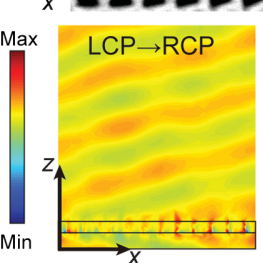

(c)
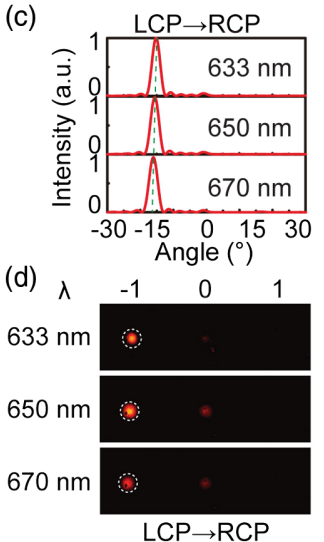
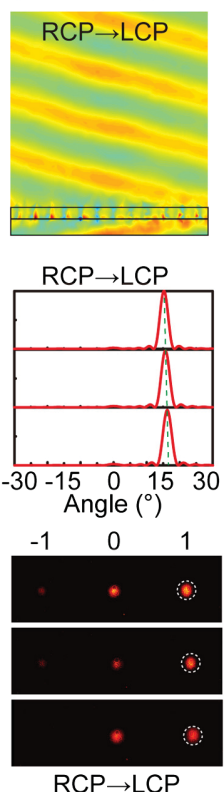

Sample 2

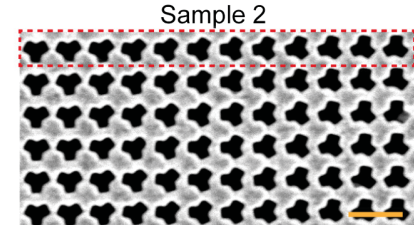

$L C P \rightarrow R C P$
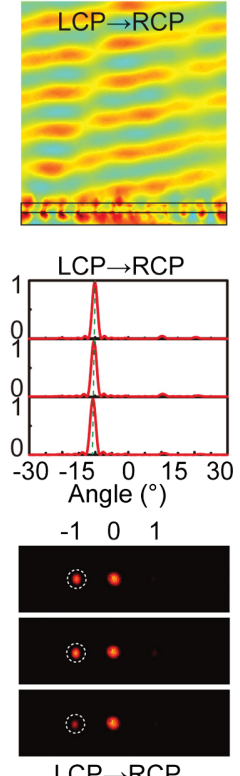

$\mathrm{LCP} \rightarrow \mathrm{RCP}$
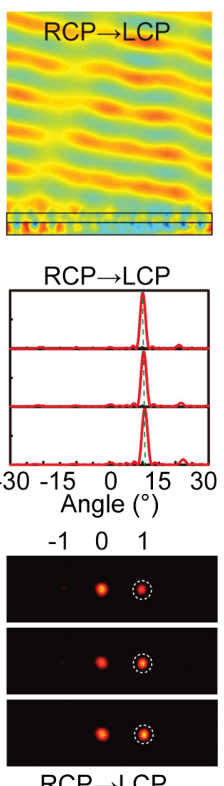

Sample 3
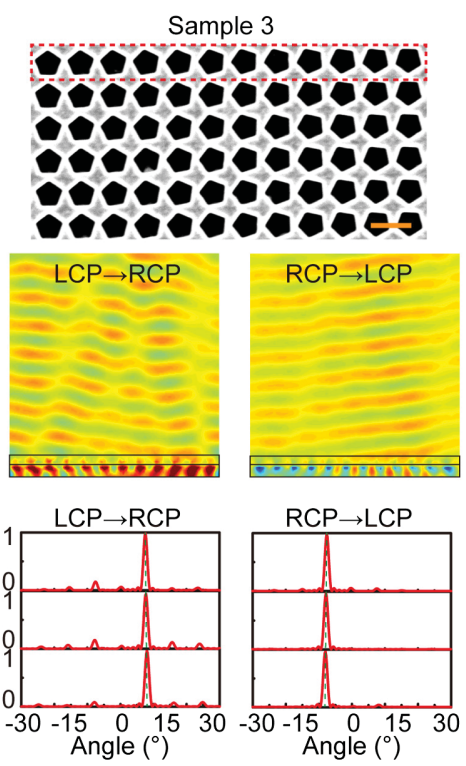

$\begin{array}{lll}-1 & 0 & 1\end{array}$

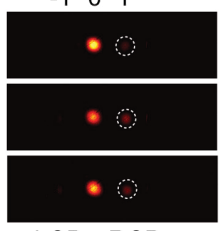

$\mathrm{LCP} \rightarrow \mathrm{RCP}$

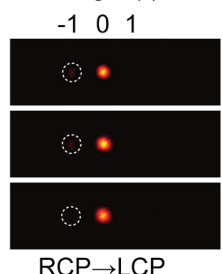

FIG. 3. (a) Scanning electron microscopy (SEM) images of the fabricated samples. Scale bars, 500 nm. All the metasurfaces have a total area of $14.4 \mu \mathrm{m} \times 14.4 \mu \mathrm{m}$. (b) Simulated $E_{\text {cross }}$ field distributions of the three metasurfaces for the incident wavelength of $633 \mathrm{~nm}$. (c) Simulated far-field intensity distributions for different incident wavelengths. The first, second, and third rows correspond to the incident wavelengths of 633, 650, and $670 \mathrm{~nm}$, respectively. (d) Measured diffraction patterns from the three samples under LCP illumination (left column) and RCP illumination (right column).

with the theoretical expectation. Subsequently, experiments were performed and the measured diffraction patterns are shown in Fig. 3(d). Under LCP or RCP illumination, the cross-polarized RCP or LCP light is diffracted to the -first or + first order for sample 1 and sample 2 , and the + first or -first order for sample 3. Owing to the imperfection of our quarter-wave plates and polarizers in the experimental setup, as well as the fabrication and measurement errors, the zeroth-order diffraction corresponding to copolarized light is not vanishing and the -first-order diffraction intensities are not fully equal to those in the first order.

To further demonstrate the large phase shifts, three vortex plates consisting of $\mathrm{C} 2, \mathrm{C} 3$, and $\mathrm{C} 5$ nanoapertures in the square lattice were designed. All the metasurfaces were created by rotating the nanoapertures $180^{\circ}$ counterclockwise in the $x-y$ plane, which could introduce a helical phase shift $\exp (i l \psi)$, creating a vortex beam carrying orbital angular momentum (OAM) [5]. Here, $l$ is the topological charge and $\psi$ is the azimuthal angle. Under LCP illumination, the generated vortex beams would possess topological charges of $l=1,3,-5$ for $\mathrm{C} 2, \mathrm{C} 3$, and C5 structures, respectively. A spherical phase was superimposed onto the helical phase to straightforwardly characterize the topological charges (see Sec. S10 of Supplemental Material for details [30]). The SEM images of the fabricated samples are depicted in Figs. 4(a) and 4(b), and the measured interference patterns are shown in Fig. 4(c). As we predicted, the rectangle-, triangle-, and pentagon-based samples can generate OAM beams with the topological charge $l=1,3$, and -5 , respectively. The measurements of triangle-based vortex plates in a broad band are also provided in Sec. S11 of Supplemental Material [30]. The nonuniformity of the intensity distributions of the triangular and pentagonal metasurfaces is resulting from the limited polarization conversion efficiency and small sample size $(R=8 \mu \mathrm{m})$, which can be ameliorated by further optimizing the structure geometry and increasing the fabrication area. For the pentagonal sample, the relatively large phase deviations shown in Fig. 1(c) would also deteriorate the performance. Moreover, because the generated topological charge is strongly dependent on the local symmetry of the metaatoms, the fabrication errors would influence the interference results especially for nanoapertures with high-order symmetries.

In summary, we have shown for the first time that rotationally symmetric meta-atoms in symmetrically incompatible lattice could induce high-order geometric phases in the linear optics regime. These surprising results 

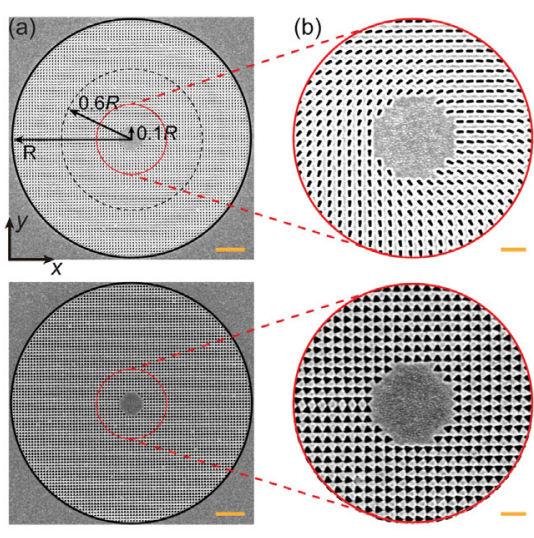

(c)
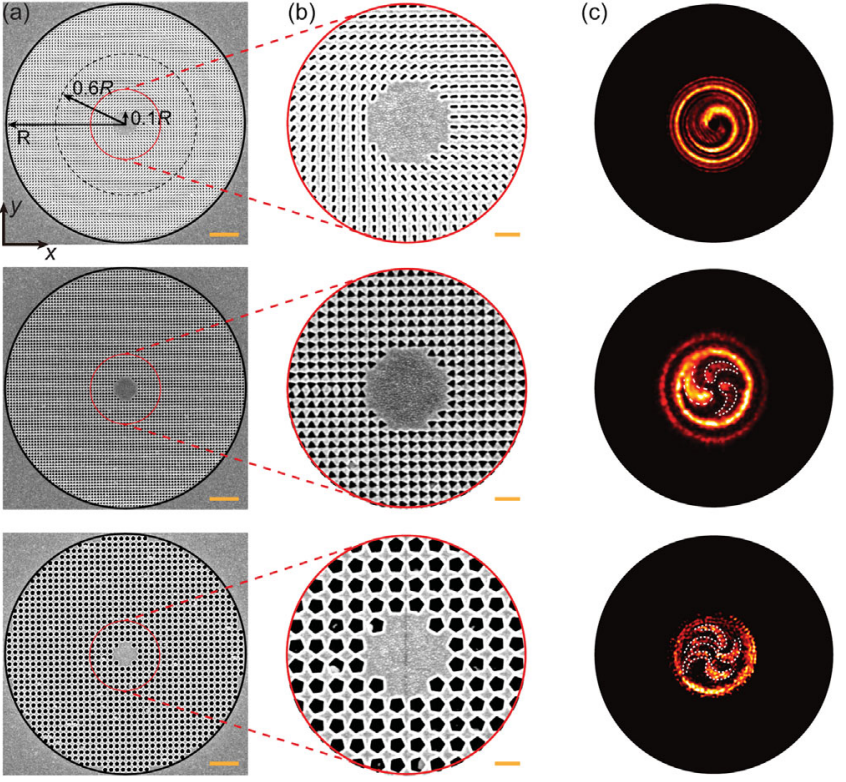

FIG. 4. (a),(b) SEM images of the fabricated vortex plates consisting of nanoapertures with $\mathrm{C} 2$ (first row), $\mathrm{C} 3$ (second row), and C5 (third row) rotational symmetry. All the samples have a radius $R=8 \mu \mathrm{m}$. Scale bars, (a) $2 \mu \mathrm{m}$, (b) $500 \mathrm{~nm}$. (c) Measured interference patterns of the vortex beams under LCP illumination with a wavelength of $633 \mathrm{~nm}$.

demonstrate that the geometric phase depends not only on the symmetry of the meta-atoms, but also on the symmetry of the lattice. For instance, C3 and C6 elements can introduce geometric phase in a square lattice, but not in a hexagonal lattice whose symmetry is consistent with that of the meta-atoms. Analogously, C4 structures have no geometric phase response in a square lattice, but geometric phases can be introduced if they are arranged in a hexagonal lattice. By controlling the local symmetry and global phase profile, angular SHEL and optical vortices generations are theoretically and experimentally demonstrated. These results may find potential applications in multifunctional light field modulation. Moreover, since the meta-atoms with high-order rotational symmetries are usually employed for harmonic generations and wave front shaping [26], they could provide a versatile platform for simultaneously tailoring linear and nonlinear optical responses.

This work was supported by the National Natural Science Foundation of China (Grant Nos. 61975210 and U20A20217).

X.X. and M.P. contributed equally to this work.

*1xg@ioe.ac.cn

[1] N. Yu and F. Capasso, Flat optics with designer metasurfaces, Nat. Mater. 13, 139 (2014).
[2] K. Y. Bliokh, F. J. Rodríguez-Fortuño, F. Nori, and A. V. Zayats, Spin-orbit interactions of light, Nat. Photonics 9, 796 (2015).

[3] Y. J. Gao, X. Xiong, Z. Wang, F. Chen, R. W. Peng, and M. Wang, Simultaneous Generation of Arbitrary Assembly of Polarization States with Geometrical-Scaling-Induced Phase Modulation, Phys. Rev. X 10, 031035 (2020).

[4] S. Sun, Q. He, S. Xiao, Q. Xu, X. Li, and L. Zhou, Gradientindex meta-surfaces as a bridge linking propagating waves and surface waves, Nat. Mater. 11, 426 (2012).

[5] N. Yu, P. Genevet, M. A. Kats, F. Aieta, J. P. Tetienne, F. Capasso, and Z. Gaburro, Light propagation with phase discontinuities: Generalized laws of reflection and refraction, Science 334, 333 (2011).

[6] G. Biener, A. Niv, V. Kleiner, and E. Hasman, Formation of helical beams by use of Pancharatnam-Berry phase optical elements, Opt. Lett. 27, 1875 (2002).

[7] D. Lin, P. Fan, E. Hasman, and M. L. Brongersma, Dielectric gradient metasurface optical elements, Science 345, 298 (2014).

[8] X. Xie, X. Li, M. Pu, X. Ma, K. Liu, Y. Guo, and X. Luo, Plasmonic metasurfaces for simultaneous thermal infrared invisibility and holographic illusion, Adv. Funct. Mater. 28, 1706673 (2018).

[9] L. Huang, X. Chen, H. Mhlenbernd, G. Li, B. Bai, Q. Tan, G. Jin, T. Zentgraf, and S. Zhang, Dispersionless phase discontinuities for controlling light propagation, Nano Lett. 12, 5750 (2012).

[10] M. Kang, T. Feng, H. T. Wang, and J. Li, Wave front engineering from an array of thin aperture antennas, Opt. Express 20, 15882 (2012).

[11] X. Ding, F. Monticone, K. Zhang, L. Zhang, D. Gao, S. N. Burokur, A. de Lustrac, Q. Wu, C.W. Qiu, and A. Alù, Ultrathin Pancharatnam-Berry metasurface with maximal cross-polarization efficiency, Adv. Mater. 27, 1195 (2015).

[12] L. Deng, J. Deng, Z. Guan, J. Tao, Y. Chen, Y. Yang, D. Zhang, J. Tang, Z. Li, and Z. Li, Malus-metasurface-assisted polarization multiplexing, Light Sci. Appl. 9, 101 (2020).

[13] S. Pancharatnam, Generalized theory of interference and its applications, Proc. Indian Acad. Sci. A 44, 398 (1956).

[14] M. V. Berry, Quantal phase factors accompanying adiabatic changes, Proc. R. Soc. A 392, 45 (1984).

[15] E. Karimi, S. A. Schulz, I. De Leon, H. Qassim, J. Upham, and R.W. Boyd, Generating optical orbital angular momentum at visible wavelengths using a plasmonic metasurface, Light Sci. Appl. 3, e167 (2014).

[16] E. Maguid, I. Yulevich, D. Veksler, V. Kleiner, M. L. Brongersma, and E. Hasman, Photonic spin-controlled multifunctional shared-aperture antenna array, Science 352, 1202 (2016).

[17] M. Pu, X. Li, X. Ma, Y. Wang, Z. Zhao, C. Wang, C. Hu, P. Gao, C. Huang, H. Ren, X. Li, F. Qin, J. Yang, M. Gu, M. Hong, and X. Luo, Catenary optics for achromatic generation of perfect optical angular momentum, Sci. Adv. 1, e1500396 (2015).

[18] K. Dou, X. Xie, M. Pu, X. Li, X. Ma, C. Wang, and X. Luo, Off-axis multi-wavelength dispersion controlling metalens for multi-color imaging, Opto-Electron. Adv. 3, 190005 (2020). 
[19] M. Khorasaninejad, W. T. Chen, R. C. Devlin, J. Oh, A. Y. Zhu, and F. Capasso, Metalenses at visible wavelengths: Diffraction-limited focusing and subwavelength resolution imaging, Science 352, 1190 (2016).

[20] S. Wang, P. C. Wu, V. C. Su, Y. C. Lai, H. C. Cheng, J. W. Chen, S. H. Lu, C. Ji, B. Xu, and C. H. Kuan, Broadband achromatic optical metasurface devices, Nat. Commun. 8, 187 (2017).

[21] L. Huang, X. Chen, H. Mhlenbernd, H. Zhang, S. Chen, B. Bai, Q. Tan, G. Jin, K. W. Cheah, and C. W. Qiu, Threedimensional optical holography using a plasmonic metasurface, Nat. Commun. 4, 2808 (2013).

[22] X. Xie, K. Liu, M. Pu, X. Ma, X. Li, Y. Guo, F. Zhang, and $X$. Luo, All-metallic geometric metasurfaces for broadband and high-efficiency wavefront manipulation, Nanophotonics 9, 3209 (2020).

[23] G. Zheng, H. Mhlenbernd, M. Kenney, G. Li, T. Zentgraf, and S. Zhang, Metasurface holograms reaching $80 \%$ efficiency, Nat. Nanotechnol. 10, 308 (2015).

[24] W. Wan, J. Gao, and X. Yang, Full-color plasmonic metasurface holograms, ACS Nano 10, 10671 (2016).

[25] M. A. Kats, P. Genevet, G. Aoust, N. Yu, R. Blanchard, F. Aieta, Z. Gaburro, and F. Capasso, Giant birefringence in optical antenna arrays with widely tailorable optical anisotropy, Proc. Natl. Acad. Sci. U.S.A. 109, 12364 (2012).

[26] G. Li, S. Zhang, and T. Zentgraf, Nonlinear photonic metasurfaces, Nat. Rev. Mater. 2, 17010 (2017).

[27] G. Li, S. Chen, N. Pholchai, B. Reineke, P. W. H. Wong, E. Y. B. Pun, K. W. Cheah, T. Zentgraf, and S. Zhang, Continuous control of the nonlinearity phase for harmonic generations, Nat. Mater. 14, 607 (2015).

[28] M. Tymchenko, J. S. Gomez-Diaz, J. Lee, N. Nookala, M. A. Belkin, and A. Alù, Gradient Nonlinear Pancharatnam-Berry Metasurfaces, Phys. Rev. Lett. 115, 207403 (2015).
[29] G. Li, L. Wu, K. F. Li, S. Chen, C. Schlickriede, Z. Xu, S. Huang, W. Li, Y. Liu, and E. Y. Pun, Nonlinear metasurface for simultaneous control of spin and orbital angular momentum in second harmonic generation, Nano Lett. 17, 7974 (2017).

[30] See Supplemental Material at http://link.aps.org/ supplemental/10.1103/PhysRevLett.126.183902 for further theoretical, simulative and experimental detail, which includes Refs. [6,7,17,31-36].

[31] X. Luo, Principles of electromagnetic waves in metasurfaces, Sci. China Phys. Mech. Astron. 58, 594201 (2015).

[32] J. B. Mueller, N. A. Rubin, R. C. Devlin, B. Groever, and F. Capasso, Metasurface Polarization Optics: Independent Phase Control of Arbitrary Orthogonal States of Polarization, Phys. Rev. Lett. 118, 113901 (2017).

[33] F. Zhang, M. Pu, J. Luo, H. Yu, and X. Luo, Symmetry breaking of photonic spin-orbit interactions in metasurfaces, Opto-Electron. Eng. 44, 319 (2017).

[34] F. Zhang, M. Pu, X. Li, P. Gao, X. Ma, J. Luo, H. Yu, and X. Luo, All-dielectric metasurfaces for simultaneous giant circular asymmetric transmission and wavefront shaping based on asymmetric photonic spin-orbit interactions, Adv. Funct. Mater. 27, 1704295 (2017).

[35] J. Samuel and R. Bhandari, General Setting for Berry's Phase, Phys. Rev. Lett. 60, 2339 (1988).

[36] J. Jin, J. Luo, X. Zhang, H. Gao, X. Li, M. Pu, P. Gao, Z. Zhao, and X. Luo, Generation and detection of orbital angular momentum via metasurface, Sci. Rep. 6, 24286 (2016).

[37] X. Luo, M. Pu, X. Li, and X. Ma, Broadband spin Hall effect of light in single nanoapertures, Light Sci. Appl. 6, e16276 (2017). 